\title{
Non-graded Base Si/Ge Heterojunction Transistor
}

\author{
H. Dombala, M. Zinaddinov, O. Kia, S. Mil'shtein
}

Advanced Electronic Technology Center, ECE Dept., University of Massachusetts Lowell, MA 01854, USA

Abstract: Innovation of Heterojunction Bipolar Transistor (HBT) technology is a major game changer in wireless communication, power amplifiers and other major fields of electronics. HBTs play a vital role in extending the advantages of silicon bipolar transistors to significantly higher levels. Research on HBT is focused on reducing cost and improving reliability. These transistors have a wide range of applications namely, digital-to-analog converters, logarithmic amplifiers, RF chip sets for CDMA wireless communication systems, and power amplifiers for cellular communications. Our study focuses on utilizing the high mobility of pure Ge instead of often-used graded Ge base. Non-grtaded Ge base enhanses carrier transport which in turn increases the gain and cut-off frequency of the HBT. We have developed a high frequency, high current gain, high power gain and less noisy heterojunction bipolar transistor operating above $100 \mathrm{GHz}$ frequency. Lattice mismatch at emitter and collector junctions is compensated by inserting SiGe buffer layers. ATLAS TCAD - SILVACO software is used for modelling of this novel device.

Keywords: $\mathrm{Si} / \mathrm{Ge}$, Heterostructured transistor, Non-graded Ge base, Lattice matching.

\section{Introduction}

Heterojunction Bipolar Transistors are manufactured using variety of semiconductor materials which include Gallium Arsenide (GaAs), Silicon (Si), Gallium nitride (GaN), and Indium Phosphide (InP). The usage of commercially available transistors with a $\mathrm{SiGe}$ graded base is a common practice in many electronic applications $^{[1,2]}$. Graded Ge base was used in design of HBT with the quantum well base ${ }^{[3]}$.

Advantages of these $\mathrm{SiGe}$ transistors ${ }^{[4]}$ over their $\mathrm{Si}$ counterparts are known, namely: very high operating frequency, low noise, high current gain and high power gain. The other crucial factors to be mentioned are low cost, ease of integration, mechanical stability, reduction in complexity of technology compared to other heterostructures mentioned above.

It is known that; Ge has mobility of electrons and diffusivity three times that of $\mathrm{Si}$. Germanium offers an electron mobility of $3900 \mathrm{~cm}^{2} \mathrm{~V}^{-1} \mathrm{~s}^{-1}$ compared to 1280 $\mathrm{cm}^{2} \mathrm{~V}^{-1} \mathrm{~s}^{-1}$ of Silicon ${ }^{[5]}$. This significantly reduces the transit time of electrons in the base and improves the gain and operating frequency of the transistor. These transistors operate at relatively high frequencies of $\mathrm{GHz}$ range, and demonstrate higher emitter injection efficiencies. Even though the base is graded and not operating at its maximum possible mobility, they operate at relatively higher frequencies. In this study, we focus on using a non-graded Ge base which enhances the mobility of carriers and improves the performance of the transistor.

We used commercial package ATLAS, a technology computer aided design (TCAD) by Silvaco for our design. Recombination models were used to define mobility of carriers, as we looked to improve the device performance based on its mobility.

\section{Design}

\subsection{Emitter and emitter-base junction}

It is a well-known tendency to increase the injection of electrons from emitter to base, for it to be much larger

Copyright (C) 2019 H. Dombala et al.

doi: 10.18686/esta.v6i1.70

This is an open-access article distributed under the terms of the Creative Commons Attribution Unported License

(http://creativecommons.org/licenses/by-nc/4.0/), which permits unrestricted use, distribution, and reproduction in any medium, provided the original work is properly cited. 
than the flow of holes from base to emitter. We used heavily doped emitter and a lightly doped base. Silicon (Si) being the emitter has the following dimensions, doping and lifetime parameters as mentioned in the Table I. The area of the emitter, $1 \mu \mathrm{m} \times 1 \mu \mathrm{m}$ is the smallest compared to other regions. We use a $0.2 \mu \mathrm{m}$ thick $\mathrm{Si}$ layer as emitter. The emitter has a $1 \mu \mathrm{m}$ long contact on the top. The intrinsic doping concentration of $\mathrm{Si}$ is $1.5 * 10^{10} \mathrm{~cm}^{-3}$.

\begin{tabular}{|c|c|}
\hline \multicolumn{2}{|c|}{ Emitter-Silicon } \\
\hline Length & $1 \mu \mathrm{m}$ \\
\hline Width & $1 \mu \mathrm{m}$ \\
\hline Thickness & $0.2 \mu \mathrm{m}$ \\
\hline Doping & $10^{19} \mathrm{~cm}^{-3}$ \\
\hline & $10^{-7} \mathrm{~s}$ \\
\hline Lifetime of electrons & $10^{-7} \mathrm{~s}$ \\
\hline Lifetime of holes & $\mathrm{r}$ \\
\hline
\end{tabular}

Table 1. Modelling parameters for emitter region

The emitter is highly doped with n-type impurities in the order of $1.0 * 10^{19} \mathrm{~cm}^{-3}$. We consider the most commonly used lifetime parameters for both electrons and holes in $\mathrm{Si}$.

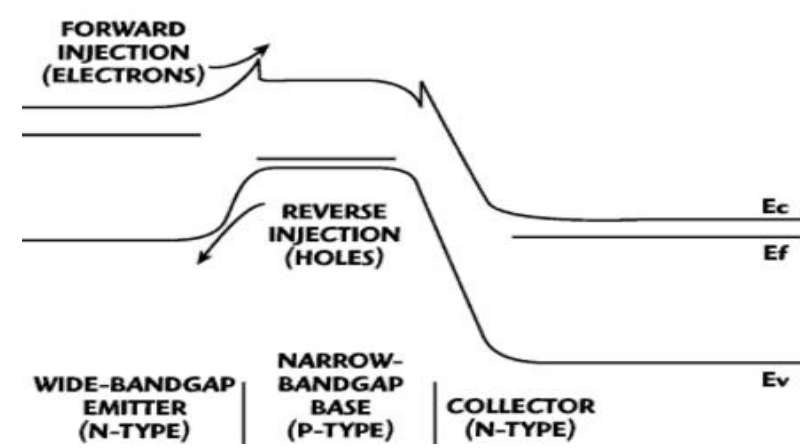

Figure 1. Band structure of n-p-n HBT design under bias with carrier flow representation

The non-graded base HBT will not allow any gradual change of energy from emitter to base. Thus, we have an abrupt junction between Emitter and Collector as seen in Figure 1. We have Si (Emitter) having a bandgap of $1.12 \mathrm{eV}$ and Ge (Base), with a bandgap of $0.67 \mathrm{eV}$.

Due to lattice mismatch between $\mathrm{Si}$ and $\mathrm{Ge}$, we faced structural discontinuity in the Emitter-Base junction, which impacts performance of the transistor. We designed a very thin SiGe buffer layer to reduce the lattice mismatch. The existence of buffer layer improves the injection of electrons into base.

\begin{tabular}{|c|c|}
\hline \multicolumn{2}{|c|}{ Emitter Base Buffer Layer } \\
\hline Length & $1 \mu \mathrm{m}$ \\
\hline Width & $1 \mu \mathrm{m}$ \\
\hline Thickness & $0.0011 \mu \mathrm{m}$ \\
\hline Doping & $1.18^{*} 10^{12} \mathrm{~cm}^{-3}$ \\
\hline Lifetime of electrons & $10^{-8} \mathrm{~s}$ \\
\hline Lifetime of holes & $10^{-8} \mathrm{~s}$ \\
\hline
\end{tabular}

Table 2. Simulation parameters for emitter-base buffer layer

The buffer layer is $0.0011 \mu \mathrm{m}(1.1 \mathrm{~nm})$ thick and is intrinsic in nature. $\mathrm{Si}_{1-\mathrm{x}} \mathrm{Ge}_{\mathrm{x}}$ buffer layer has $\mathrm{x}=0.4$. The lifetime of electrons and holes, in Table $\mathbf{2}$ are considered based on the alloy composition.

\subsection{Base region}

The base region stores the minority carrier charge when the transistor is in the active mode. For a $n-p-n$ transistor, the stored electron charge in the base, $\mathrm{Q}_{\mathrm{n}}$, and the collector current $i_{c}$ have the following relationship

$$
Q_{n}=\tau_{F} i_{c}
$$

where $\tau_{\mathrm{F}}$ is a device constant with the dimension of time. It is called the forward base transit time and gives the average time that a charge carrier (electron) takes to cross the base.

As Equation (1) applies only for large signals, the small-signal diffusion capacitance $\mathrm{C}_{\mathrm{de}}$ can be given as,

$$
\begin{aligned}
C_{d e}= & \frac{d Q_{n}}{d V_{B E}}=\frac{d i_{c}}{d V_{B E}} \\
& \therefore \quad C_{d e}=\tau_{F} g_{m}=\tau_{F} \frac{I_{c}}{V_{T}}
\end{aligned}
$$

where $\mathrm{I}_{\mathrm{C}}$ is the dc collector bias current at which the transistor is operating.

Emitter-Base junction has an area of $0.2 \mu \mathrm{m} \times 1 \mu \mathrm{m}$. We use a 0.04 um (400 Angstrom) thick Ge layer as Base. The intrinsic doping concentration of $\mathrm{Ge}$ is $1.73 \mathrm{e}+13$ $\mathrm{cm}^{-3}$. The base is lightly doped with p-type impurities in the order of $4.0 \mathrm{e}+16 \mathrm{~cm}^{-3}$. We consider the most commonly used lifetime parameters from Table 3, for both electrons and holes in Ge.

\begin{tabular}{|c|c|}
\hline \multicolumn{2}{|c|}{ Base } \\
\hline Length & $2.2 \mu \mathrm{m}$ \\
\hline Width & $1 \mu \mathrm{m}$ \\
\hline Thickness & $0.04 \mu \mathrm{m}$ \\
\hline Doping & $4.0^{*} 10^{16} \mathrm{~cm}^{-3}$ \\
\hline $\begin{array}{c}\text { Lifetime of } \\
\text { electrons }\end{array}$ & $4.0^{*} 10^{-5} \mathrm{~s}$ \\
\hline Lifetime of holes & $4.0^{*} 10^{-5} \mathrm{~s}$ \\
\hline
\end{tabular}

Table 3. Simulation parameters for base region 
The thickness of the base almost allows us to be in the quantization range (around 250-300 Angstrom). Our device shows the best performance at 400 Angstrom.

\subsection{Collector and collector-base junction}

Collector has the largest area out of all three regions. Larger area compensates the heat dissipated in this region due to inward flow of large number of charge carriers.

The depletion capacitance of the reverse biased Collector-Base junction is given by

$$
C_{\mu}=\frac{C_{\mu 0}}{\left(1+\frac{V_{C B}}{V_{0 c}}\right)^{m}}
$$

where $C_{\mu}$ is the depletion capacitance; $C_{\mu 0}$ is the value of $\mathrm{C}_{\mu}$ at zero voltage; $\mathrm{V}_{\mathrm{CB}}$ is the magnitude of the CBJ reverse-bias voltage, $\mathrm{V}_{0 \mathrm{c}}$ is the $\mathrm{CBJ}$ built-in voltage and $\mathrm{m}$ is its grading coefficient.

Equations (2-4) addresses values of junction capacitances. These capacitances define cut-off frequency of the transistor.

The area of the collector, $2.2 \mu \mathrm{m} \times 1 \mu \mathrm{m}$ is the largest compared to other regions. We use a $0.2 \mu \mathrm{m}$ thick Si layer as collector. The collector has a $2.2 \mu \mathrm{m}$ long contact at the bottom. The intrinsic doping concentration of $\mathrm{Si}$ is $1.0 \mathrm{e} 10 \mathrm{~cm}^{-3}$. The emitter is moderately doped with n-type impurities in the order of $3.8 \mathrm{e} 17 \mathrm{~cm}^{-3}$. We consider the most commonly used lifetime parameters from Table IV, for both electrons and holes in Si.

The discontinuity can be seen at the junction formed between the base and collector like the emitter-base junction. This reduces the gain, frequency and other important performance parameters in the transistor. There is a need to match the lattice between the heterostructures. This is done by adding a buffer layer as discussed in section (II-a).

The buffer layer is $0.0011 \mu \mathrm{m}(1.1 \mathrm{~nm})$ thick and is intrinsic in nature. $\mathrm{Si}_{1-\mathrm{x}} \mathrm{Ge}_{\mathrm{x}}$ has a $\mathrm{x}$-composition of 0.8 . Electrons from Ge base will have a smooth transition to $\mathrm{Si}$ if the buffer layer has parameters close to Ge, thus $80 \% \mathrm{Ge}$ is used in the buffer. The lifetime of electrons and holes, in Table 5, are considered based on the alloy composition.

\begin{tabular}{|c|c|}
\hline \multicolumn{2}{|c|}{ Collector Base Buffer Region } \\
\hline Length & $2.2 \mu \mathrm{m}$ \\
\hline Width & $1 \mu \mathrm{m}$ \\
\hline Thickness & $0.0011 \mu \mathrm{m}$ \\
\hline Doping & $2.31 \mathrm{e}+13 \mathrm{~cm}^{-3}$ \\
\hline Lifetime of electron & $1.0 \mathrm{e}-08 \mathrm{~s}$ \\
\hline Lifetime of hole & $1.0 \mathrm{e}-08 \mathrm{~s}$ \\
\hline
\end{tabular}

Table 5. Simulation parameters for collector-base buffer layer

\section{Simulation results}

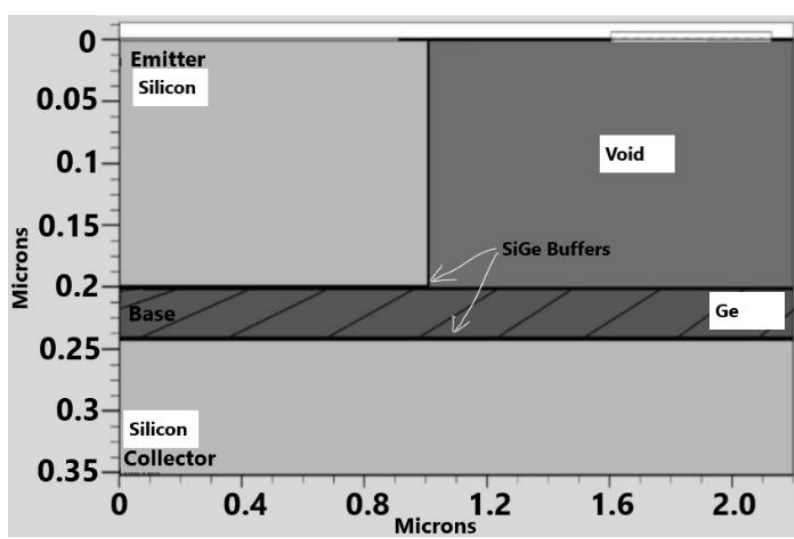

Figure 2. Structure of Non-graded base SiGe HBT

The structure in Figure 2 shows the SiGe HBT designed under this study. This $2 \mathrm{D}$ plot represents the thickness of the various regions in micrometer along the length of the transistor.

The Gummel plot for our transistor with collector at $2 \mathrm{~V}$ and various base voltages is seen in Figure 3. The collector current at various base currents can be observed and thus current gain factor, $\beta$, can be computed using Gummel plot.

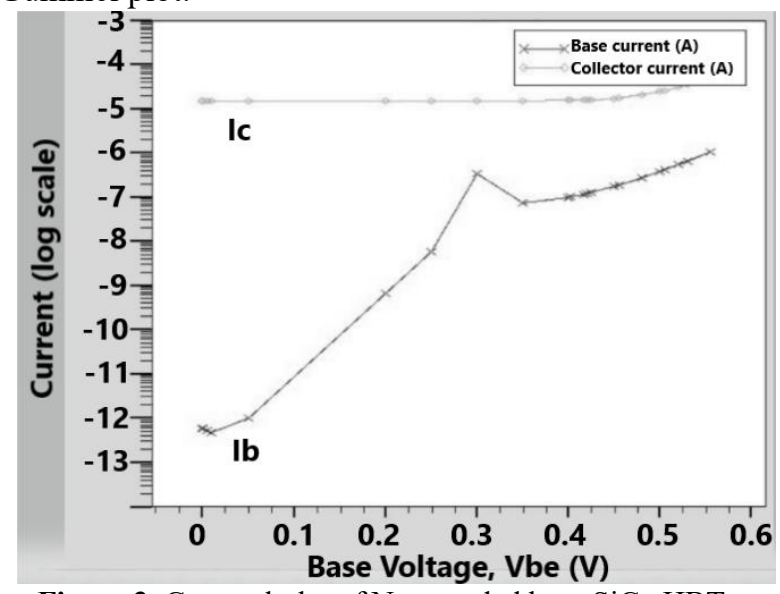

Figure 3. Gummel plot of Non-graded base SiGe HBT 


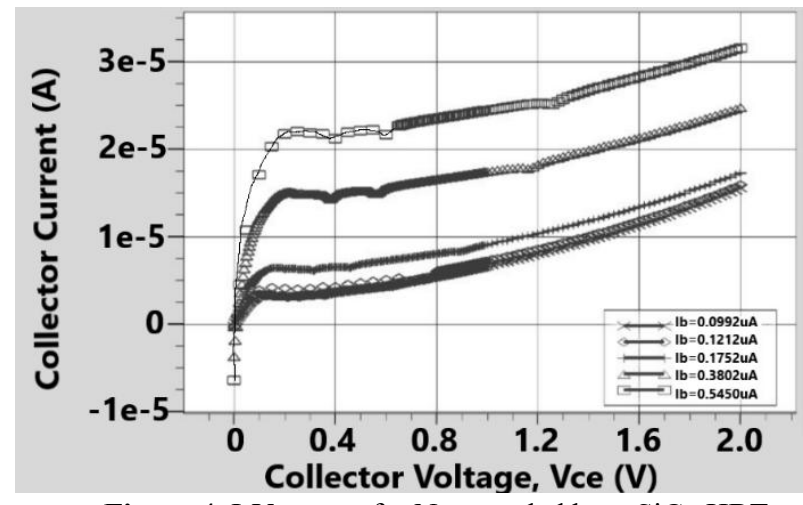

Figure 4. I-V curves for Non-graded base SiGe HBT

\begin{tabular}{|c|c|c|}
\hline $\mathbf{V}_{\mathbf{b e}}$ & $\mathbf{I}_{\mathbf{b}}$ & $\mathbf{I}_{\mathbf{c}}$ \\
\hline $0.40 \mathrm{~V}$ & $0.0992 \mu \mathrm{A}$ & $15.56 \mu \mathrm{A}$ \\
\hline $0.42 \mathrm{~V}$ & $0.1212 \mu \mathrm{A}$ & $15.93 \mu \mathrm{A}$ \\
\hline $0.45 \mathrm{~V}$ & $0.1752 \mu \mathrm{A}$ & $17.26 \mu \mathrm{A}$ \\
\hline $0.50 \mathrm{~V}$ & $0.3802 \mu \mathrm{A}$ & $24.78 \mu \mathrm{A}$ \\
\hline $0.52 \mathrm{~V}$ & $0.5450 \mu \mathrm{A}$ & $31.58 \mu \mathrm{A}$ \\
\hline
\end{tabular}

Table 4. $I_{c} v s V_{c e}$ for different $I_{b}$ extracted for various $V_{b e}$

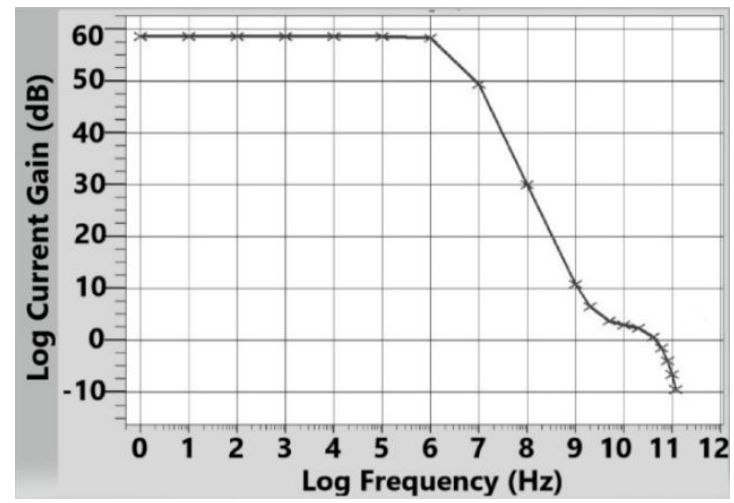

Figure 5. Current gain (dB) of Non-graded base SiGe HBT

For a transistor with common emitter configuration, the output current $\left(\mathrm{I}_{\mathrm{c}}\right)$ is plotted against output voltage $\left(\mathrm{V}_{\mathrm{ce}}\right)$ for various base biasing as seen in Figure 4. The values for various curves are tabulated in Table 6.

The AC analysis of our design is carried out by biasing the collector at $2 \mathrm{~V}$ for various base biasing voltages. This analysis at various frequencies yields the plot in Figure 5. The maximum unity gain cut-off frequency, $\mathrm{f}_{\mathrm{t}}$, is obtained and used for the performance characteristics of HBT.

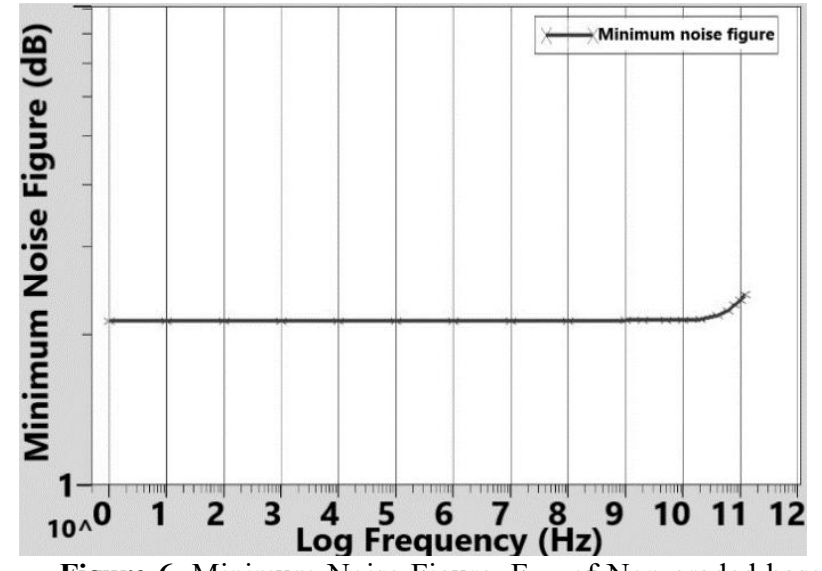

Figure 6. Minimum Noise Figure, $F_{\text {min }}$ of Non-graded base SiGe HBT

Noise figure ${ }^{[7]}$ is a figure-of-merit that describes the amount of excess noise present in a system. Minimizing the noise in the system reduces system impairments and improves the performance of the device ${ }^{[8]}$. It is one of the most important parameters for radio communications applications when assessing sensitivity. As seen in Figure VI, AC analysis of our device gives us the Minimum noise figure of around $2 \mathrm{~dB}$ along the operating range of frequencies.

S21 represents the power transferred from input port to output port ${ }^{[9]}$. In general, $\mathrm{AC}$ analysis of the device should be done to obtain the $\mathrm{S}$ parameters. Figure 7 shows the Power transfer capability of our HBT in terms of $\mathrm{S} 21$. The maximum oscillation frequency, $f_{\max }$ is determined by S21 vs Frequency plot

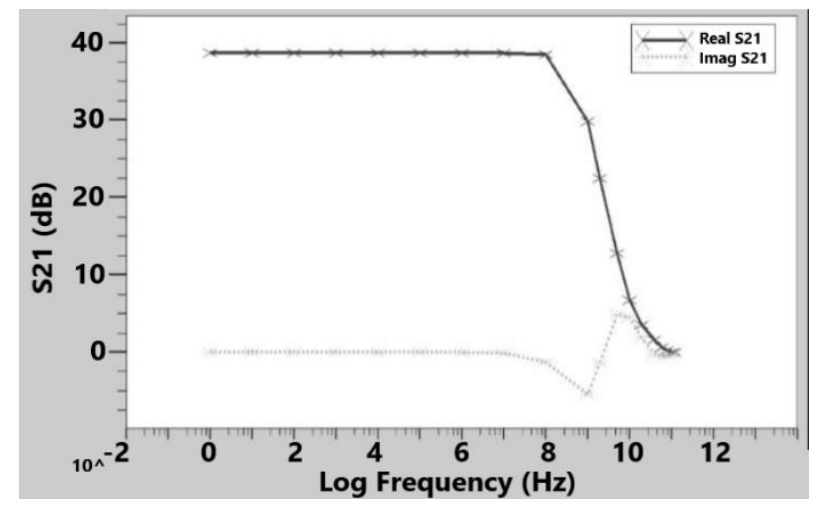

Figure VII. S21 of Non-graded base SiGe HBT

Summary of Performance of Non-graded base SiGe HBT

Table 7 summarizes the performance characteristics of our Non-graded base SiGe HBT. Section IV analyzes the performance parameters and gives a comparative study of our HBT with commercially available Graded base SiGe HBTs. 


\begin{tabular}{|c|c|}
\hline \multicolumn{2}{|c|}{ Simulation Results } \\
\hline Ib (max) & $0.5450 \mu \mathrm{A}$ \\
\hline Ic (max) & $31.58 \mu \mathrm{A}$ \\
\hline $\begin{array}{c}\text { Trans-conductance } \\
\text { gain }\end{array}$ & $34 \mathrm{~mA} / \mathrm{V}$ \\
\hline Current gain & $59 \mathrm{~dB}$ \\
\hline S21 & $15.91 \mathrm{~dB}$ \\
\hline $\begin{array}{c}\text { Minimum Noise } \\
\text { Figure }\end{array}$ & $2 \mathrm{~dB}$ \\
\hline $\mathbf{f}_{\mathbf{t}}$ & $50 \mathrm{GHz}$ \\
\hline $\mathbf{f}_{\max }$ & $110 \mathrm{GHz}$ \\
\hline
\end{tabular}

Table 7. Summary of extracted simulation results

\section{Conclusion}

We discussed in this study, modelling and simulation results of a non-graded base $\mathrm{Si} / \mathrm{Ge} \mathrm{HBT}$. Ability to create heterostructure $\mathrm{Si} / \mathrm{Ge}$ bipolar transistor with non-graded Ge base carries apparent mobility advantage over conventional $\mathrm{Si} \mathrm{BJT}$ as well as over graded Ge base devices. Ease of integration with already existing silicon wafer technology gives $\mathrm{Si} / \mathrm{Ge}$ HBTs priority in integrated design. Applications for a transistor with high gain and very high frequency of operation in addition to low noise, are many. Companies like IBM Microelectronics, Intel, and Infineon technologies, are spending billions of dollars on research on $\mathrm{SiGe}$ technology ${ }^{[10]}$.

Looking at the research trends on SiGe technology ${ }^{[11]}$, IBMs graded epi-base SiGe HBT works with the following key outputs, $\mathrm{f}_{\mathrm{t}}\left(\right.$ at $\mathrm{V}_{\mathrm{cb}}=1 \mathrm{~V}$ ) $=47 \mathrm{GHz}, \mathrm{f}_{\max }$ (at $\left.\mathrm{V}_{\mathrm{bc}}=1 \mathrm{~V}\right)=65 \mathrm{GHz}$, Beta $(\beta)\left(\right.$ at $\left.\mathrm{V}_{\mathrm{be}}=0.72 \mathrm{v}\right)=100$.

A 0.5 um SiGe-HBT technology ${ }^{[12]}$ with a trapezoidal Ge-profile of $8 \%$ has a $f_{t}\left(\right.$ at $\left.V_{c b}=1 \mathrm{~V}\right)=32 \mathrm{GHz}, f_{\max }$ (at $\left.\mathrm{V}_{\mathrm{bc}}=1 \mathrm{~V}\right)=45 \mathrm{GHz}$, Beta $(\beta)\left(\right.$ at $\left.\mathrm{V}_{\mathrm{cb}}=0\right)=43$.

Quick reference to processing steps show that the Reduced Pressure Chemical Vapor Deposition (RPCVD), high strained, Ge-graded (15.5\% - 0\%) SiGe film, used as a base of the HBT (Heterojunction Bipolar Transistor) developed in $0.35 \mu \mathrm{m}$ SiGe BiCMOS process technology ${ }^{[13]}$, works with DC characteristics of $\beta=150$ and high-frequency performance, $\mathrm{f}_{\mathrm{t}}=67 \mathrm{GHz}$.

The $\mathrm{Si} / \mathrm{Ge}$ heterojunction bipolar transistor process using differential epitaxy and in situ phosphorus-doped poly-Si emitter ${ }^{[14]}$ at very low thermal budget is designed. A very high current gain of almost 2000 and cut-off frequency of $62 \mathrm{GHz}$ were achieved for a uniform $12 \%$ Ge profile.
The Non-graded base Si/Ge HBT discussed in this paper performs well on both $\mathrm{DC}$ and AC parameters. A current gain of $891(59 \mathrm{~dB})$, S21 of $39(15.91 \mathrm{~dB})$, Maximum unity current gain frequency, $\mathrm{f}_{\mathrm{t}}=50 \mathrm{GHz}$ and a maximum oscillation frequency of $110 \mathrm{GHz}$, was observed. A unique distinction of our device from all the comparable works is that the high frequency is associated with high gain. This improvement in almost all parameters can be credited to the pure Ge channel used in the design. Using different composition SiGe buffer layers at both junctions play an important role in achieving these results. High performance parameters coupled with easy integration with existing technology is the next step to be taken towards future electronics evolution.

\section{Acknowledgements}

The authors appreciate participation of Mr. K. Chatzopoulos in the early stage of this study.

\section{References}

1. https://nepp.nasa.gov/DocUploads/0624ECCF-180F 4C0AA3ACF526CFFBCD66/pub_hbt_paper.pdf

2. http://www.wiasberlin.de/people/glitzky/HTML/proj ect2.html

3. Sam Mil'shtein, Samed Halilov, John Palma, "SiGe HBT with Quantum Well Base", Intern. Confer, Phys. Semicond.-2014, p. 15, Aug. 2014

4. http://www.microwavejournal.com/articles/2659-sig e-tansistor-technology-for-rf-applications

5. http://www.ioffe.ru/SVA/NSM/Semicond/

6. Adel S. Sedra et al., "Microelectronic Circuits Theory and Applications", Oxford University Press, P.159 to P.298.

7. http://www.keysight.com/upload/cmc_upload/All/M aury-WebEx-NewUltra-FastNoiseParameterSystem. $\mathrm{pdf} ? \& \mathrm{cc}=\mathrm{US} \& \mathrm{lc}=\mathrm{eng}$

8. Donald A. Neamen, "Semiconductor Physics and Devices- Basic Principles", Mcgraw Hill Companies Inc, P.491 to P.558

9. http://www.antenna-theory.com/definitions/sparamet ers.php

10. R. Szweda, "Silicon Germanium Materials and Devices - A Market and Technology Overview to 2006”, Elsevier Science

11. D. C. Ahlgren, M. Gilbert, D. Greenberg, S. J. Jeng, 
J. Malinowski, D. Nguyen-Ngoc, K. Schonenberg, K. Stein, R. Groves, K. Walter, G. Hueckel, D. Colavito, G. Freeman, D. Sunderland, D. L. Harame, and B. Meyersori, IBM Microelectronics Division, Hopewell Junction, New York. *IBM Research Division, Yorktown Heights, New York. "Manufacturability Demonstration of an Integrated SiGe HBT Technology for the Analog and Wireless Marketplace", Electron Devices Meeting, 1996. IEDM '96., International

12. Harame. K. Schonenberg, M. Gilbert, et al., IBM Res. Div., Yorktown Hts., NY, USA, *IBM Microelectronics Division, Ifopewell Junct., NY, EE Dept Auburn IJniv., Auburn, AL, Analog Devices, Greensboro, NC, Analog Devices, Wilmington, MA, "A $200 \mathrm{~mm}$ SiGe-HBT technology for wireless and mixed-signal applications", 1994 IEEE International Electron Devices Meeting.

13. Jing Zhang, Yonghui Yang, Guangbing Chen, Yuxin Wang, Dongbing Hu, Kaizhou Tan, Wei Cui, Zhaohuan Tang, National Laboratory of Analog Integrated Circuits, Chongqing, China, "A Ge-Graded SiGe HBT with $\beta>100$ and $\mathrm{fT}=67$ GHz", World Journal of Engineering and Technology, 2015.

14. J.V. Grahn*, H. Fosshaug, M. Jargelius, P. JoÈ nsson, M. Linder, B.G. Malm, B. Mohadjeri, J. Pejnefors, H.H. Radamson, M. SandeÂn, Y.-B. Wang, G.Landgren, M. Ostling, "A low-complexity 62-GHz fT SiGe heterojunction bipolar transistor process using di erential epitaxy and in situ phosphorus-doped poly-Si emitte emitter at very low thermal budget", Solid-State Electronics 44 (2000) 549-554. 